\title{
The future of CABN — A conceptual triad: Psychological theory, neurobiological approaches, computational methods
}

\author{
Marie T. Banich ${ }^{1}$
}

Published online: 19 January 2016

(C) Psychonomic Society, Inc. 2016

With the advent of this volume, I, along with a new board of associate editors, am privileged to be fully taking over the helm of Cognitive, Affective, \& Behavioral Neuroscience $(C A B N)$ from the former editor-in-chief, Deanna Barch, and her board. Where last year's volume contained articles reviewed and accepted by Deanna and her team, this volume represents the first set of papers to be published from our editorial tenure. We are lucky in that the former editors have done an outstanding job in creating a unique niche for $C A B N$ - skillfully shepherding the journal to publish sophisticated research within the subfields covered by the journal, as well as high-quality synthetic theoretical reviews. As the saying goes, "If it ain't broke, don't fix it." In that spirit, we intend to maintain the journal's overall focus on publishing across the range of cognitive, affective, and behavioral neuroscience, and to continue to publish articles that examine psychological processes in both typical populations and those with relevant psychiatric, neurological, and associated syndromes. As I have experienced in my own work, the interchange between basic and clinical science can be highly productive. Psychological theories can provide insights into disruptions in clinical populations, while patterns of performance and neural function in clinical populations can help to confirm or guide the development of psychological theories. Moreover, cross-fertilization between work performed in humans and in animals can be similarly informative, especially with the advent of new techniques that allow neural systems to be more directly manipulated in animals. These approaches are likely to only increase the degree to which animal models will

Marie T. Banich

Marie.Banich@colorado.edu

1 University of Colorado Boulder, Boulder, CO, USA aid in building our understanding of the neural mechanisms underlying behavior.

In building upon the firm foundation of the previous editorial teams, the goal of the $C A B N$ editorial board will be, quite simply, to make $C A B N$ the journal in which to publish if one has (a) a strong model or hypothesis regarding psychological processes (cognitive and/or affective) that (b) is tested through approaches linked to brain and neural processes in (c) a statistically or computationally rigorous and/or groundbreaking manner. In our increasingly multidisciplinary and multifaceted scientific world, we strongly believe there is an important place for a journal that specifically serves to bring such strands of the scientific endeavor together in an integrated manner. Tests of psychological theory are increasingly being informed by both neuroscientific evidence and the more sophisticated quantitative tools provided by statistical modeling and machine learning. Yet, as is always the case with multidisciplinary work, it is hard to have both breadth and depth. We wish $C A B N$ to showcase those who defy such a stereotype by demonstrating that hypothesis-driven, multifaceted research can be both deep and wide. The different and often complementary approaches provided by such integrated research endeavors can allow for a synergy and a leap forward in understanding not found in studies of more limited scope. In addition, such multifaceted approaches provide a richness of explanation that is important for scientific progress. Our goal is that when you read through an issue of $C A B N$, you will learn something new and interesting, be provided with an insight or vantage point that differs from the usual, and know that the ideas presented and the data to back them up have been rigorously examined from a variety of angles, using converging approaches.

For our part, we want to help bring such research to the field by making $C A B N$ 's editorial process as quick, fair, and seamless as possible, while at the same time ensuring that it is 
thoughtful and thorough. To help ensure a quick review, we have a two-step review process. All submissions are viewed by myself, and often by an associate editor, to determine whether they indeed fall within the journal's scope and meet a variety of criteria, including whether the work tests a clear hypothesis, makes a contribution and is of interest to the field, and has methods, sample and effect sizes that are stringent enough to ensure that the reported research will be competitive for space in the journal. In this manner, all submissions have a quick initial vetting. Also to aid in a quick review process, we will continue the tradition of usually making a final decision after the first resubmission. Although this latter procedure can put some burden on authors to make substantial changes from their initial submission, we believe it also enables authors to do the most possible to improve their manuscripts, and leads to faster decision times.

To ensure a thorough and thoughtful review, once a submission has been deemed to meet the journal's initial criteria, it is then assigned to either myself or one of the associate editors. I have been very fortunate that an excellent international team of five associate editors have agreed to serve the journal. Each of their own programs of research exemplifies the type of work we strive to publish in $C A B N$, and their expertise spans the many different subfields of neuroscience reflected in the journal's title and mission. They are backed up by a board of consulting editors notable for their expertise, breadth of knowledge, and geographical expanse, as well as by the generous support of many scholars across the field who serve as reviewers. You can expect from all of us on the editorial board a careful weighing of the feedback provided to you as an author, rather than just a quick tally of how many "accept," "revise and resubmit," or "reject" evaluations we have received. And you can also expect a clear action letter that highlights those aspects of a study's approach, methods, or conclusions that need to be reconsidered, as well as sug- gestions about how best to present the study to readers, so as to make the questions and hypotheses salient and the outcome clear. Editorial decisions are based on many criteria, including not only the reviews themselves, but also the judgment of members of the associate editorial board as to whether a study is well-designed and has clear conclusions, adds important knowledge to the field, and is of broad interest to the readers of $C A B N$ and others across the field. While I know that sometimes aspects of a manuscript review can seem tedious, the process almost always results not only in better science, but in better presentation of the work as well. No matter how brilliant a study is, unless it is presented in an accessible and understandable manner, its impact will be limited. As such, our team of associate editors are actually partners to help ensure that your research has the highest impact possible.

I am very excited to be at the helm of $C A B N$, not only because of the journal's stellar reputation, but also because, with the vantage point of having been in the field for numerous decades, I can honestly say that the present day is one of the most stimulating times in my experience. What makes the present so exciting is the way in which the different subfields covered by the journal are coming together to answer questions about the organization and structure of our thoughts and feelings, and the way in which this endeavor is being aided by ever-developing, paradigmshifting neurobiological and computational methods. It is my hope that $C A B N$ will serve to aid in this burgeoning scientific endeavor by demonstrating the power of the triad of an explicit and testable psychological theory, the additional insights and constraints provided by neurobiological approaches, and the formal predictive and evaluative power supplied by computational approaches. Should you have any suggestions of how to improve the journal, concerns, criticisms, or even compliments, please do feel free to contact me at Marie.Banich@colorado.edu. 